\title{
Note of Appreciation to Paper Reviewers
}

The quality of the technical journal Geosynthetics International, and hence its reputation as the premiere peer-reviewed journal on geosynthetics and related topics, depends on the dedication of the many reviewers who donate their time and expertise to assess submissions to the journal. The Editor and Chair of the Editorial Board would like to thank the following individuals for completing manuscript reviews for the papers that appeared in Volume 22 of the journal, or which were declined for publication in 2015.

$$
\text { R. J. Bathurst J. P. Giroud }
$$

H. Abuel-Naga

A. H. Aydilek

H. Bannour

M. W. Bo

M. Bouazza

L. Briancon

F. Canestrari

G. Cascante

J. Castro

D. Cazzuffi

J.-C. Chai

K. T. Chang

M. Chappel

A. Chinkulkijniwat

H. Choi

J. Chu

J. Clancy

N. C. Consoli

N. Correia

I. P. Damians

A. De

K. Deb

G. di Emidio

A. Diambra

A. Edincliler

M. El-Naggar

D. Elton

S. J. Feng

L. Festugato

P. J. Fox

G. Fownes

R. Gilbert

J. P. Giroud

I. Gogo-Abite
I. Gonzalez-Torre

J. L. Hanson

K. Hatami

G. Heerten

M. Heibaum

A. Helwa

W. Hornsey

S. Horpibulsuk

C. C. Huang

T. Hutchinson

M. B. Jaska

M. Kachan

V. Kaliakin

D. Kim

G. Koerner

W. Kongkitkul

J. Koseki

A. M. Krishna

C. K. Lau

C. Lawson

B. Leshchinsky

M.-H. Li

C.-N. Liu

K. Liu

S. Liyanapathirana

M. R. Madhav

G. Madhavi Latha

F. Mazzieri

R. P. McAffee

J. McCartney

R. L. Michalowski

Y. Miyata

I. M. Moore

D. Negussey
A. Neto

E. M. Palmeira

E. Pasquini

I. Peggs

S. Perkins

M. Pinho-Lopes

R. H. Plaut

F. H. M. Portelinha

M. Prezzi

A. J. Puppala

S. Restall

R. K. Rowe

J. Scheirs

P. Simms

T. Stark

A. W. Take

C.-S. Tang

B. Tanyu

R. Thiel

N. Touze-Foltz

A. C. Trandafir

S. Vanapalli

A. Vega-Zamanillo

C. S. Vieira

P. Villard

B. V. S. Viswanadham

H. Wallbaum

E. Williams

M. Xiao

K. Yasuhara

T. M. Yee

J.-H. Yin

Y. W. Yoon 